\title{
Monitoring neurogenesis in the cerebral cortex: an update
}

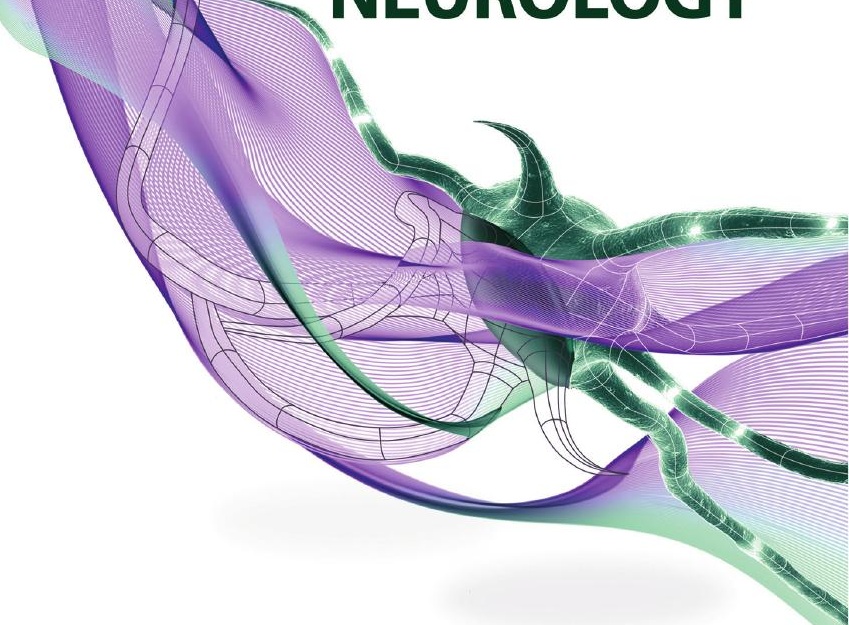

Maria Pia Postiglione ${ }^{1} \&$ Simon Hippenmeyer*,1

ABSTRACT: The cerebral cortex, the seat of our cognitive abilities, is composed of an intricate network of billions of excitatory projection and inhibitory interneurons. Postmitotic cortical neurons are generated by a diverse set of neural stem cell progenitors within dedicated zones and defined periods of neurogenesis during embryonic development. Disruptions in neurogenesis can lead to alterations in the neuronal cytoarchitecture, which is thought to represent a major underlying cause for several neurological disorders, including microcephaly, autism and epilepsy. Although a number of signaling pathways regulating neurogenesis have been described, the precise cellular and molecular mechanisms regulating the functional neural stem cell properties in cortical neurogenesis remain unclear. Here, we discuss the most up-to-date strategies to monitor the fundamental mechanistic parameters of neuronal progenitor proliferation, and recent advances deciphering the logic and dynamics of neurogenesis.

\section{Medscape: Continuing Medical Education Online}

This activity has been planned and implemented in accordance with the Essential Areas and policies of the Accreditation Council for Continuing Medical Education through the joint sponsorship of Medscape, LLC and Future Medicine Ltd. Medscape, LLC is accredited by the ACCME to provide continuing medical education for physicians.

Medscape, LLC designates this journal-based CME activity for a maximum of 1 AMA PRA Category 1 Credit $(s)^{\mathrm{TM}}$. Physicians should claim only the credit commensurate with the extent of their participation in the activity.

All other clinicians completing this activity will be issued a certificate of participation. To participate in this journal CME activity: (1) review the learning objectives and author disclosures; (2) study the education content; (3) take the post-test with a $70 \%$ minimum passing score and complete the evaluation at www.medscape.org/journal/fnl; (4) view/print certificate.

RELEASE DATE: 5 JUNE 2014; EXPIRATION DATE: 5 JUNE 2015

IIST Austria (Institute of Science \& Technology Austria), Am Campus 1, A-3400 Klosterneuburg, Austria

*Author for correspondence: simon.hippenmeyer@ist.ac.at 


\section{LEARNING OBJECTIVES}

Upon completion of this activity, participants should be able to:

- Describe recent techniques for monitoring neurogenesis, based on a review

- Discuss recent advances in understanding the dynamics of neurogenesis

- Assess future directions for research and clinical implications regarding neurogenesis

Financial \& competing interests disclosure

Editor: Elisa Manzotti, Publisher, Future Science Group.

Disclosure: Elisa Manzotti has disclosed no relevant financial relationships.

CME author: Laurie Barclay, MD, Freelance writer and reviewer, Medscape, LLC.

Disclosure: Laurie Barclay, $M D$, has disclosed no relevant financial relationships.

Author \& credentials: Maria Pia Postiglione, PhD, Institute of Science and Technology Austria, Klosterneuburg, Austria.

Disclosure: Maria Pia Postiglione, PhD, has disclosed no relevant financial relationships.

Simon Hippenmeyer, PhD, Institute of Science and Technology Austria, Klosterneuburg, Austria.

Disclosure: Simon Hippenmeyer, $P h D$, has disclosed the following relevant financial relationships: received grants from: Institute of Science and Technology Austria institutional funds and by funding from the People Programme (Marie Curie Actions) of the European Union's Seventh Framework Programme (FP7/2007-2013) under REA grant agreement FP7-CIG618444.

No writing assistance was utilized in the production of this manuscript.

\section{KEYWORDS}

- cerebral cortex

- lineage $\bullet$ lissencephaly

- microcephaly • mitotic spindle $\bullet$ mosaic analysis with double markers

- neurogenesis

\section{Overview: cerebral cortex development}

One of the most striking evolutionary features underlying the unique cognitive human abilities is the emergence of an exceedingly enlarged cerebral cortex. The origin of the neocortex can be traced back to reptiles, which show for the first time a laminar, although simple, organization of telencephalic projection neurons [1,2]. A progressive increase in the numbers of cortical neurons, size and shape of the neocortex resulted in the emergence of the highly folded (gyrencephalic) mammalian brain found in the majority of primates and humans [3-5]. The human cerebral cortex is composed of an extraordinary number of neurons and glia cells that originate from a variety of progenitor stem cells located in the ventricular (VZ) and subventricular (SVZ) zones (Figure 1) $[5,6]$. The identification and classification of progenitor stem cells that produce the different types of neurons in the CNS has a long history that started more than a century ago. In 1887 , His introduced the concept of two distinct progenitor types, one each for neurons and glial cells, on the basis of histological observations of mitotic figures in the VZ of human embryos. He suggested that these proliferating cells were germinal 'Keimzellen', producing one daughter cell that remained in the $\mathrm{VZ}$, and a postmitotic 'neuroblast' that migrated away and became a neuron. The nonproliferating or quiescent cells within the $\mathrm{VZ}$ on the other hand were believed to constitute a syncytium of spongioblasts that would later give rise to glia cells [7]. The concept of His has been challenged by Vignal, Schaper and Koelliker, who suggested that the neuroblasts and spongioblasts were alternative forms of the same progenitor cell, while moving from the apical to the basal side of the VZ, depending on the phase of the cell cycle [8-10]. Their notion was later supported by Sauer who also described the phenomenon of interkinetic nuclear migration (IKNM) in the VZ. During IKNM, mitotic progenitor cell nuclei display asynchronous but alternate movements depending on their cell cycle phase. While in the G1 phase, the nucleus migrates to the basal end of the progenitor, proceeds through the $S$ phase and moves back to the apical side in G2 to eventually complete mitosis [11-13]. Nevertheless, the concept proposed by His remained prominent until live imaging at the individual progenitor cell level demonstrated that radial glia progenitors (RGPs) account for the generation of all excitatory projection neurons in the cerebral cortex $[14,15]$. Cortical 


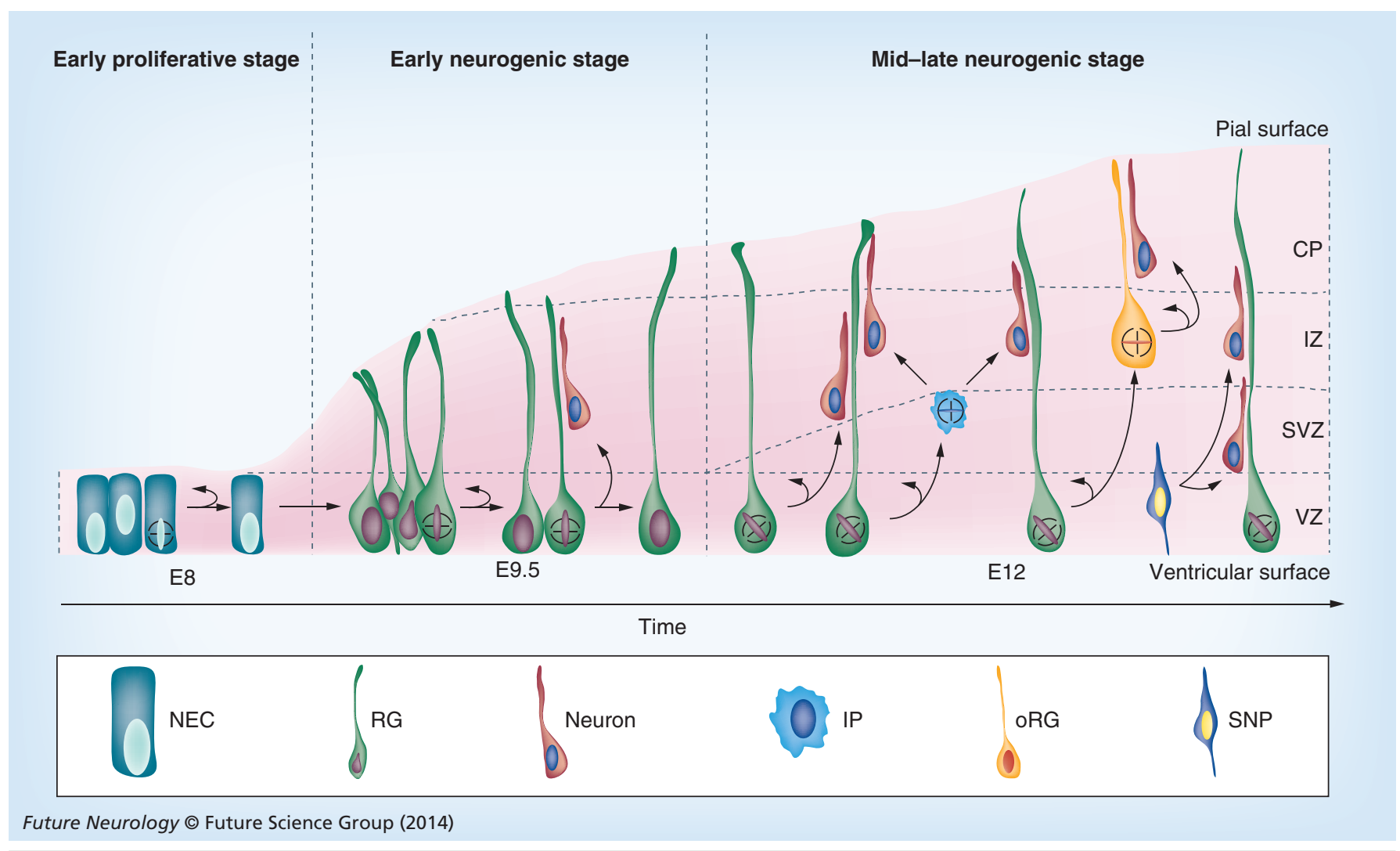

Figure 1. Neurogenesis in the mouse cortex. NECs initially divide symmetrically to expand their pool and progressively transform into radial glia progenitor (RGP) cells. RGPs divide symmetrically, and asymmetrically to produce neurons that migrate radially to form the CP. At earlier stages, RGPs divide asymmetrically to produce neurons directly, then switch to indirect neurogenesis through IPs or oSVZ oRGs. oRGs divide asymmetrically to produce neurons in mice, or transient amplifying cells in humans. IPs mostly populate the SVZ while oRGs translocate into the IZ for neurogenesis. SNPs are located in the VZ, where they divide symmetrically to produce neurons. CP: Cortical plate; IP: Intermediate progenitor; IZ: Intermediate zone; NEC: Neuroepithelial cell; oRG: Outer radial glia; RG: Radial glia; SNP: Short neural precursor; SVZ: Subventricular zone; VZ: Ventricular zone.

projection neurons are produced in consecutive waves during embryogenesis. They assemble in an inside-out fashion whereby earlier generated neurons populate innermost layers, and later generated neurons radially migrate along the elongated basal glial processes of RGPs to settle in more superficial layers in the developing cortex [16,17]. Severe neurological disorders, including megalencephaly, microcephaly and lissencephaly, but also more subtle neurodevelopmental disorders, such as schizophrenia and autism, can result if neurogenesis is impaired or neuronal migration is disrupted in human [18-22]. Excitatory cortical projection neurons represent approximately $80 \%$, and inhibitory interneurons approximately $20 \%$ of all neurons in the cerebral cortex [23]. Cortical interneurons are generated by progenitors in the ventral telencephalon, and migrate tangentially to the developing cortical plate [24-26]. Interestingly, interneuron progenitors in the ventral ganglionic eminences also display a morphological RGP fate character with an extended basal process [27-29], albeit dorsal and ventral RGPs express distinct sets of fate determining transcription factors [30,31]. During the last decade, besides RGPs a variety of distinct progenitors (Figure 1), including intermediate progenitors (IPs), short neural precursors (SNPs), transient amplifying progenitors (TAPs) and outer SVZ (oSVZ) progenitors (outer radial glia cells [oRGs]) have been discovered $[3,5,6,32]$. The broader criteria for a coarse classification of neuronal progenitors include the site of mitosis, cell polarity, molecular makeup, mode of division and proliferative capacity $[3,5,6,32]$. Although it is currently not clear how many different progenitor types exist at all, and how they holistically orchestrate neurogenesis, several key questions emerge regarding the precise numbers and distinct neuron 
classes that are produced by the entire progenitor community but also individually by each progenitor during cortical neurogenesis. For instance, which cellular and molecular programs dictate the quantitative neuron output in distinct progenitor types? On the qualitative level, what are the precise neuronal lineages that are generated from individual progenitor cells? Also, more generally, how do progenitor stem cells shape and influence the fate of distinct lineages, which may on their part contribute to establish specificity of neuronal connectivity in defined cortical circuits? In order to begin to address these fundamental questions it is important to precisely monitor neurogenesis at the individual progenitor level, and to functionally dissect the signaling pathways that regulate neuronal progenitor identity and neurogenic dynamics. This article will include an update on the currently available approaches that enable the tracing of neurogenesis at high resolution and also discuss recent advances that provide novel mechanistic insight into the cellular and molecular principles of neurogenesis.

\section{Monitoring neurogenesis}

\section{- Neuron birth dating}

Major advances in tracking neurogenesis became possible once tracers like tritiated thymidine $\left(\left[{ }^{3} \mathrm{H}\right]\right.$-thymidine) were introduced. Tritiated thymidine is a radiolabeled deoxynucleoside that is incorporated into the DNA during replication in cell proliferation and remains in the nuclei of dividing cells as a permanent marker [33]. Birth-dating experiments, in which $\left[{ }^{3} \mathrm{H}\right]$-thymidine was administrated to rodents at different times during brain development, showed that neurons that are born at the same time occupy the same layer, thereby building up the cortex in an 'inside-first, outside-last' fashion [16]. Tritiated thymidine was substituted over the time by nonradioactive and/or halogenated thymidine analogs like 5-bromo-2'-deoxyuridine, 5-ethynyl-2-deoxyuridine, 2'-deoxy-5-iodouridine or 5-chloro-2'-deoxyuridine, which can be visualized by staining with specific antibodies [34-36]. Thymidine analogs in combination with markers of cell proliferation, such as $\mathrm{Ki} 67$, allow quantitative assessment of the postmitotic neuron production rate and numbers of cycling progenitors. In effect, the rate of cell cycle exit and neurogenesis can be determined by quantification of the proportions of progeny from mitoses exiting the cell cycle (quit fraction) or continuing to proliferate across defined temporal windows [37].

A genetic approach has also been developed to selectively detect neurogenic progenitors during cortex development. In particular, expression of GFP from the Tis 21 locus in mice specifically labels neurogenic progenitors and permits dynamic live imaging analysis [38]. Tis21-GFP mice have been successfully used to isolate molecular markers for neurogenic progenitors and in combination with cumulative DNA labeling using thymidine analogs to determine temporal cell cycle parameters in distinct progenitor populations [39,40]. An elegant contemporary method allows the direct tracking of cell cycle changes in real time and thus assay cell proliferation in single progenitor cells. This method, called fluorescent ubiquitination-based cell cycle indicator (FUCCI) (Figure 2A), couples dual color imaging with distinct cell cycle progression phases [41]. FUCCI allows the precise distinction between the G1 and the S/G2/M phases by expression of two distinct (red and green) fluorescent probes in living cells. The FUCCI probes mark nuclei in G1 phase in red and those in S/G2/M phases in green. Thus, FUCCI permits in principle the live tracking of the dynamic cell cycle oscillations during IKNM in the ventricular zones of the developing brain and thus the 4D monitoring of neurogenesis [41] (Figure 2). FUCCI technology has recently been further extended and two optimized probes have been introduced in the genomic Rosa26 locus [42] to also enable conditional and cell type-specific expression of the FUCCI probes by using different Cre recombinase drivers [43]. Altogether, FUCCI technology holds the potential to systematically determine cell cycle parameters and neurogenic dynamics in genetically distinct neuronal progenitors in vivo and in real time by using live imaging.

\section{- Monitoring neurogenesis by lineage tracing}

Whether different classes of neurons are generated from the same progenitor cell type, or from distinct fate-restricted progenitors is a major open issue $[30,44]$. In other words, does one type of progenitor sequentially generate a series of different neurons by changing or adjusting its neurogenic potential over time? Or do different progenitors generate only one and always the same type of neurons during their neurogenic phase? This topic has been intensely 


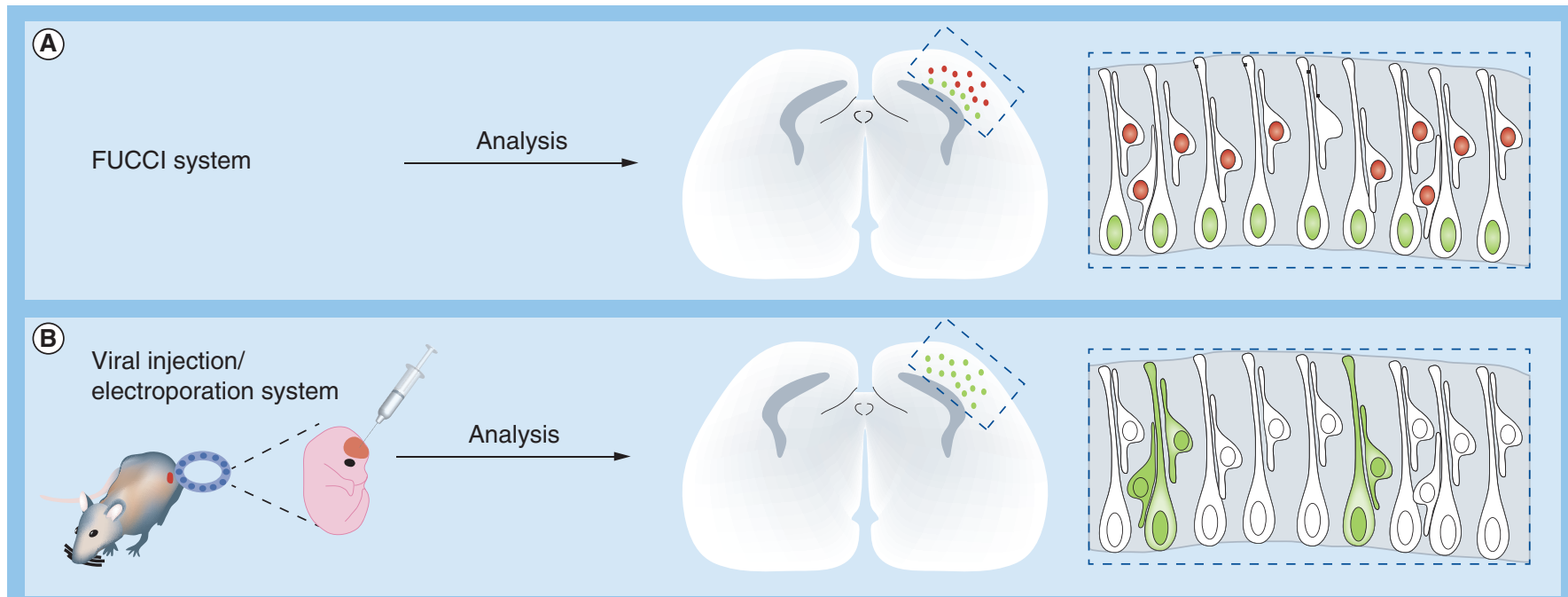

(c)

Conditional reporter system

Analysis
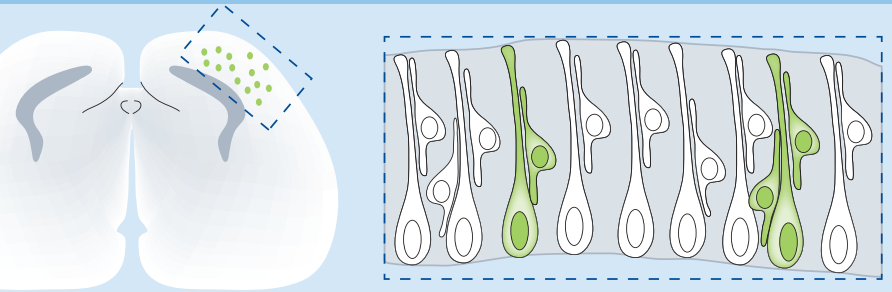

(D)

Confetti system
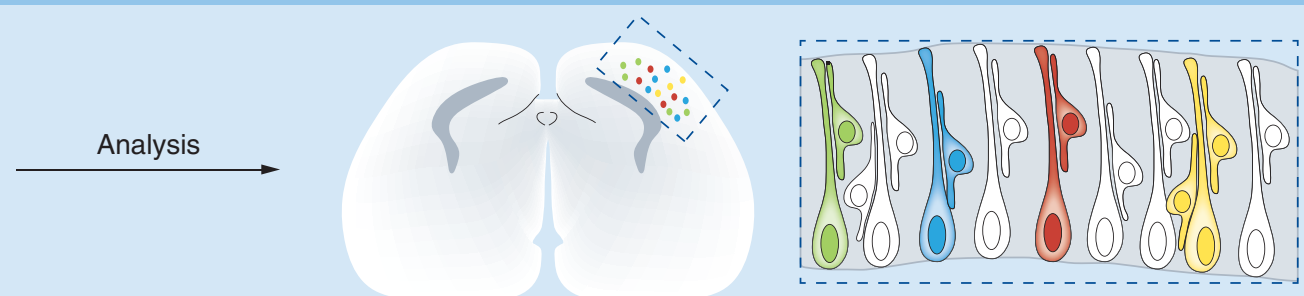

(E)

MADM system

Analysis
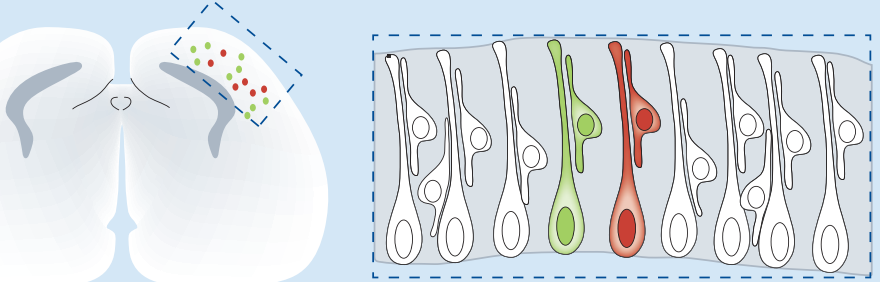

Future Neurology @ Future Science Group (2014)

Figure 2. Lineage tracing systems for monitoring neurogenesis. Lineage tracing systems and expected outcomes in assays of cortical neurogenesis. (A) FUCCl-mediated tracking of neural progenitor cell cycle dynamics. FUCCI probes can distinctly mark nuclei of dividing progenitors in G1 phase (red) and those in S/G2/M phases (green). The red marker is retained in differentiating neurons as illustrated. (B) A virus or a plasmid can be used as vehicles to express a genetic cell marker (e.g., enhanced green fluorescent protein) in neural progenitors via in utero injection into the lateral ventricle and/or electroporation in mouse embryos. Low titers of virus (or concentration of electroporated plasmids) allow the labeling of sparse clones. (C) Sparse CreER-mediated recombination of a reporter allele in cortical progenitors and their clonal progeny. (D) Sparse induction of Confetti by using CreER-mediated recombination in neural progenitors may generate several individual but distinctly labeled (green, red, cyan or yellow) clones. (E) MADM-based two-color labeling of cortical clones. FUCCl: Fluorescent, ubiquitination-based cell cycle indicator; MADM: Mosaic analysis with double markers.

studied in the cerebral cortex by using diverse lineage tracing (determination of progenitor cell division pattern and distribution of clonally related neurons that build up the cortex) assays.

Historically, lineage tracing methods including transplantation approaches, chimera generation, 
retrovirus infection, electroporation or recombination-based fate mapping allowed mostly post hoc examination of clones [45-48]. Here, we focus on a set of conditions that also enable the monitoring of neurogenesis in real time by live imaging.

\section{Lineage tracing by using retroviruses}

In order to pursue high resolution single cell lineage tracing, progenitor stem cells should be marked in a sparse but permanent manner. In the most optimal case, the marker will be transferred to the whole lineal progeny and even after numerous rounds of cell division still robustly mark all daughter cells. Retroviruses encoding markers such as GFP or LacZ have been widely used to trace the progeny generated by a single neural progenitor cell (Figure 2B). Low titers of retrovirus were used to infect embryonic mouse and rat cortices by in utero injection into the ventricles at different developmental stages. Initially, these paradigms were applied to study the composition of neural progenitor derived lineages, and the distribution of clonally related cells at postnatal stages [49-51]. A real breakthrough was achieved when GFP expressing retrovirus infections of neuronal progenitor cells was coupled with live imaging protocols to monitor neurogenesis by individual progenitors in real time $[15,52,53]$. These studies represent true landmarks since they for the first time demonstrated that RGPs are mitotically active throughout neurogenesis and produce neurons either directly or indirectly via intermediate progenitors (see also below). In addition to in utero application of retroviruses into the ventricle of developing embryos, a technically somewhat similar approach can also be used: in utero electroporation of plasmids encoding fluorescent markers such as GFP [54] (Figure 2B). If carried out at low concentration and if plasmid DNA is carefully titrated, clonal labeling may be obtained and in combination with live imaging can reveal the dynamics of neurogenesis. In utero electroporation also has the advantage that multiple plasmids may be coelectroporated and gene knockdown experiments in dividing progenitors may provide mechanistic insight on a molecular functional level [55].

\section{Lineage tracing by using}

recombinase-mediated reporter expression

A versatile approach which enables dynamic lineage tracing is based upon the use of the Cre
recombinase/LoxP system. Ideally, Cre recombinase expression is controlled by a progenitor cell type-specific promoter. Cre recombinase-mediated excision of a transcriptional Stop sequence flanked by LoxP sites (commonly denominated as LSL), promotes marker gene expression from distinct reporters within genetically defined cell populations (Figure $2 C \& 3 A$ ) [42,56-58]. The modification of the Cre recombinase to render it inducible (CreER versions) greatly improved the analysis of neurogenesis and more generally the study of brain development and function [59]. CreER is a fusion of Cre recombinase with the ligand binding domain of the estrogen receptor (ER) and inducible by the estrogen analog tamoxifen (TM) [60]. The high efficiency of the CreER recombinase combined with conditional reporter alleles can generate large numbers of labeled progenitor cells and their downstream lineages. Thus one can analyze neurogenesis on a population level. However, by titrating the dose of TM, one can, in principle, also sparsely label clonal progenitor lineages in the developing cortex. Neurogenesis of individual progenitors can be assayed by using high resolution live imaging protocols. An important restriction of TM-mediated CreER induction is that the maximum TM dose that can be applied to pregnant female mice is limited (in order to avoid high abortion rate or infanticide). Alternatively, low titer infection with Cre recombinase-expressing retroviruses or electroporation of plasmids encoding Cre recombinase can also be used to sparsely induce marker gene expression and even at the clonal density (Figure 2B \& 3A) [52,54]. Such efforts allowed, for instance, the unprecedented lineage analysis of RGPs generating cortical interneurons in the medial ganglionic eminence $[28,29]$. Both studies used the expression of Cre recombinase under the control of a transcription factor, $\mathrm{Nkx} 2.1$ expressed in the interneuron progenitor cells. In one case [28], the Nkx2.1-Cre driver was first crossed to a reporter mouse conditionally encoding the viral receptor. Next, lowtiter retrovirus encoding GFP was applied by in utero intraventricular injection. The approach used by Ciceri and colleagues employed similar in utero retrovirus application but here the expression of the virally encoded marker was conditional and dependent upon Cre expression driven from the Nkx2.1 locus [29]. Brown and colleagues then used dynamic live imaging of labeled RGPs generating cortical interneurons and analyzed their neurogenic properties. 


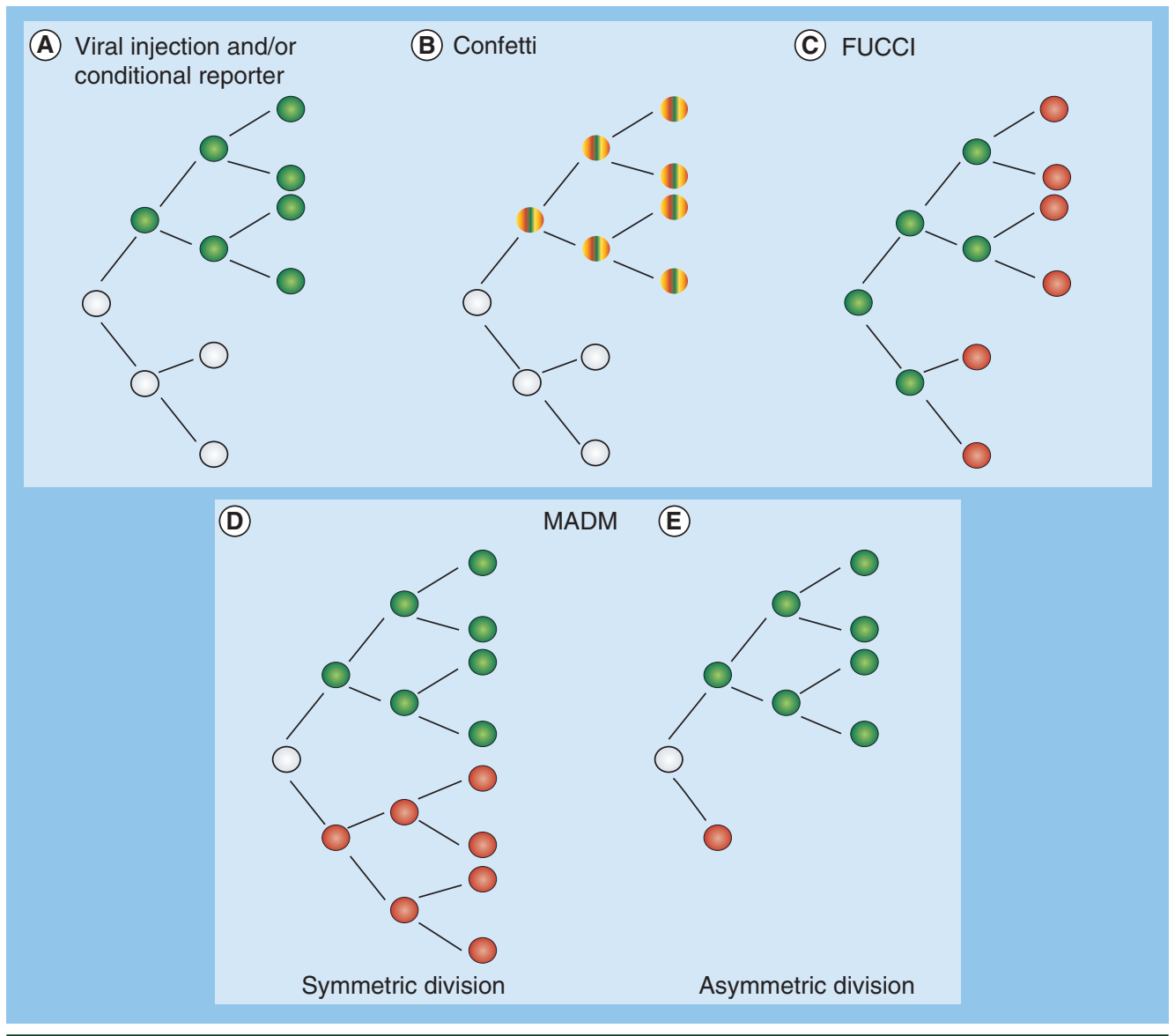

Figure 3. Comparison of expected cortical progenitor lineage trees. (A) Viral injection, plasmid electroporation or CreER-mediated sparse recombination of a reporter allele generates monocolor lineages. (B) CreER-mediated sparse recombination of the Confetti cassette can generate individual clones in distinct colors (presented as four color rainbow). (C) FUCCl activation generates cells that express green or red markers depending on the cell cycle stage. Progenitors that are in the G1 phase of the cell cycle (and postmitotic neurons) will be marked in red, while cells in the S/G2/M phase will appear in green. (D \& E) MADM can generate two daughter cells expressing either the green or red marker. The mode of progenitor division can be inferred by comparison of the numbers of neurons in red and green, respectively. (D) Upon symmetric division, the green and red progeny of a MADM clone is equal in size (green/red ratio is 1). (E) Asymmetric progenitor divisions generate clones with different red and green cell numbers (green/red ratio $>1$ in this example).

FUCCl: Fluorescent, ubiquitination-based cell cycle indicator; MADM: Mosaic analysis with double markers.

Remarkably, these interneuron-producing RGPs closely resembled the RGPs in the dorsal telencephalic VZ generating excitatory projection neurons. In effect, RGPs located in the medial ganglionic eminence also exhibited IKNM, divided at the surface of the $\mathrm{VZ}$ and generated interneurons by asymmetric divisions [28].

The recombinase-based techniques described so far permit the tracing of one or just a few genetically defined progenitor lineages in one color. It would however be ideal to label multiple lineages at the same time and in distinct colors. The
Brainbow strategy [61] can in principle accommodate such multicolor labeling of distinct progenitor lineages. Brainbow enables the conditional but stochastic expression of multiple fluorescent proteins from a single transgene, using Cremediated excision between pairs of incompatible lox sites. Combination of multiple copies of the Brainbow transgene allows the multiple fluorescent proteins to recombine in different ways and could, in principle, result in cell labeling with up to approximately 100 different fluorescent color hues [61]. Transgenic Brainbow cassettes 
were recently inserted into the genomic Rosa26 locus (also known as Confetti) [62]. Confetti was originally generated to trace intestinal stem cell progeny but can in principle be used to trace any progenitor lineage including the neuronal lineages by using appropriate Cre recombinase drivers (Figure 2D \& 3B) [63].

Lineage tracing by using mosaic analysis with double markers

One of the most classic approaches for neurogenic lineage tracing involves the creation of genetically mosaic animals where two or more subpopulations of dividing progenitor stem cells have distinct genotypes. Several mosaic labeling systems have been established by using genetic approaches in distinct organisms [46,64-66]. Here, we focus on the mosaic analysis with double markers (MADM) technique in mice $[64,67]$. For MADM, two reciprocally split marker gene (GFP-tdT) cassettes are introduced at identical genomic loci on homologous chromosomes [67-69]. These split marker genes can be reconstituted in a LoxP/Cre recombinase-dependent manner in mitotically active neuronal progenitor stem cells via interchromosomal recombination. As a consequence of a MADM event, the two progeny of a neuronal progenitor cell are labeled in two distinct colors, GFP and red tdT, respectively. Since interchromosomal (trans) recombination rates are much (up to 100-1000-times) less frequent than intrachromosomal or cis recombination, as described in the above sections, MADM can generate very sparse individual progenitor cell clones in combination with temporally TM-inducible CreER drivers. Thus, in the most optimal scenario, a single MADM event can generate one twocolored clone originating from a single neuronal stem cell (Figure 2E). In combination with $4 \mathrm{D}$ live imaging, MADM, can in principle provide unambiguous information on the birth dates of individual progenitor clones and their precise cell division patterns (i.e., whether they divide symmetrically or asymmetrically) [69] (Figure 3D \& E). An added MADM feature is the possible introduction of gene mutations allowing clonal two-color labeling with concomitant genetic manipulation. As such, these MADM applications lead to genetic mosaics with wildtype daughter cells labeled with one color (e.g., red) and homozygous mutant siblings with the other (e.g., green) in an unlabeled heterozygous environment. In summary, MADM can provide a quantitative optical readout of the proliferation mode (symmetric vs asymmetric) of progenitors at the single cell level (Figure 3D \& E) and thus permit the determination of the developmental progenitor potential in situ. Future functional MADM analysis of candidate genes controlling the balance between expansive symmetric and neurogenic asymmetric progenitor cell division also promise the systematic dissection of molecular pathways regulating the division mode of neuronal progenitor stem cells in time and space.

\section{Cortical stem \& progenitor cells dynamically orchestrate cortical neurogenesis}

The early embryonic neuroepithelium is composed of neuroepithelial stem cells from which all subsequent neural progenitor stem cells and their neuron lineages derive. In this section we will describe recent advances of how distinct progenitor classes orchestrate neurogenesis and point to open questions that will be discussed in the perspective below.

\section{- Neuroepithelial cells}

In all mammals the entire CNS is derived from a single densely packed pseudostratified layer of highly polarized neuroepithelial cells (NECs) (Figure 1). The pseudostratified appearance of the neuroepithelium is a reflection of the IKNM [12]. NEC polarity is essential for neuroepithelial organization, maintenance and homeostasis [70] . Furthermore, polarized distribution of cell fate determinants in NECs appears to control the fine balance between symmetric and asymmetric progenitor divisions [70]. Such a balance is critical for the generation of the appropriate number of the more specialized RGPs and thus represents a fundamental requirement for producing the correct number of neurons in the adult cortex. In the initial stages of neurogenesis, NECs arrange the mitotic spindle parallel (division plane perpendicular) to the VZ and divide mostly symmetrically, thereby expanding the progenitor pool [32]. Perturbation of spindle anchoring to the lateral NEC walls leads to randomization of spindle orientation, precocious generation of neurons and apoptosis [71]. Lineage tracing experiments demonstrated that many NECs are also capable of dividing asymmetrically to produce neurons, before they generate RGPs [72-74]. Starting from E9 in mouse, NECs transform into radial glial cells [75]. It is currently, however, not entirely clear if this process involves an asymmetric NEC 
division or merely a cellular and/or molecular rearrangement within the NECs. Thus, it will be important in the future to determine the precise cellular and molecular mechanisms controlling the transition from NECs to RGPs.

\section{- Radial glia progenitor cells}

The NEC to RGP transition is associated with changes in morphology, gene expression, and mode of division [75]. At the onset of neurogenesis, RGPs progressively switch from a symmetric proliferative to an asymmetric neurogenic mode of division that give rise not only to neurons, but also to other neural progenitor populations including IPs and oRGs (Figure 1) [14,15,27,53,76-78] . The precise molecular mechanisms controlling the fate of the RGP daughter cells are currently unclear but subject of extensive research. Lineage tracing coupled with live imaging and loss of function approaches as described above, hold the greatest potential to unravel the precise principles of asymmetric RGP divisions. Several models of how asymmetric RGP division can be achieved have been proposed. These models include parameters such as cleavage plane orientation, mitotic spindle-size asymmetry, dynamic distribution of polarity cues, differential inheritance of the basal process, cell cycle length and the asymmetric inheritance of the centrosome and/or ciliary membrane [79-82]. Regardless of the precise mechanisms in each model (except possibly the cell cycle length model), either cell fate determinants or entire organelles (e.g., centrosome, basal process) are asymmetrically segregated prior to mitosis and differentially inherited by the two distinct daughter cells. The 'fate determinant' segregation can rely entirely on intrinsic mechanisms or may depend on extrinsic factors that generate local differences inside the receiving cell. Similar to NECs, RGPs divide mostly symmetrically at early stages of neurogenesis (E9-E11 in mouse) with the mitotic spindle oriented in parallel to the VZ [75]. Perturbation of the spindle orientation at these stages by loss of function of for instance the evolutionarily conserved Lis1-complex leads to precocious RGP-mediated neurogenesis and depletion of the RGP pool $[22,55,71,83]$. Thus, the control of spindle orientation is critical in the first phases of neurogenesis to keep the balance between symmetric proliferative and asymmetric neurogenic radial glial cells divisions. As the rate of neurogenesis increases during mid corticogenesis, the balance shifts towards a predominant asymmetric mode of division with an increase in the production of neurons either by direct or indirect neurogenesis via IPs and oRGs (Figure 4A) [5]. Recent evidence suggests that the orientation of the mitotic spindle can have a direct influence on the fate of the daughter cell produced from RGPs [77,84]. In effect, artificial alteration of spindle orientation in dividing RGPs towards a more oblique mode of division by overexpression of the adaptor protein mouse INSC causes an increase in oRG production by RGPs in the IZ/SVZ of the mouse developing cortex (Figure 4A) [84]. Albeit the orientation of the mitotic spindle could fulfill a direct and instructive role in determining the cell fate of the daughter cells produced by RGPs, it has been shown that spindle-size asymmetry also coincides with asymmetric RGP division (Figure 4B) [79]. Neurons appear to originate preferentially from the larger, and the self-renewing RGP from the smaller spindle pole. Mechanistically, components of the planar cell polarity signaling pathway seem to regulate spindle-size asymmetry [79]. During RGP mitosis, the centrosome (i.e., both of its two centriols) is duplicated and it has been suggested that the asymmetric inheritance of the 'older' mother centrosome versus the 'younger' daughter centrosome could play an important role in asymmetric RGP division (Figure 4C) [85]. Although the precise mechanisms remain to be determined, dividing RGPs might employ asymmetric centrosome inheritance as a means of cellular memory whereby the mother centrosome is preferentially inherited by the self-renewing RGP and the daughter centrosome by the differentiating sister cell [85]. Furthermore, the ciliary membrane attached with the mother centriole is endocytosed at the onset of RGP mitosis and inherited by the self-renewing RGP. It has been proposed that the centrosomal association of the ciliary membrane could act in an instructive way to promote expedited ciliogenesis in the nascent RGP and thereby retaining important stem cell fate-promoting signaling from the ventricular cerebrospinal fluid (Figure 4C) [81,86].

\section{- IPs \& SNPs}

IPs are located predominantly in the VZ/SVZ, express the transcription factor Tbr2 (also know as Eomes) and are present throughout cortical neurogenesis $[87,88]$. Single cell gene expression profiling and lineage tracing indicate that two (morphologically distinct) subtypes 


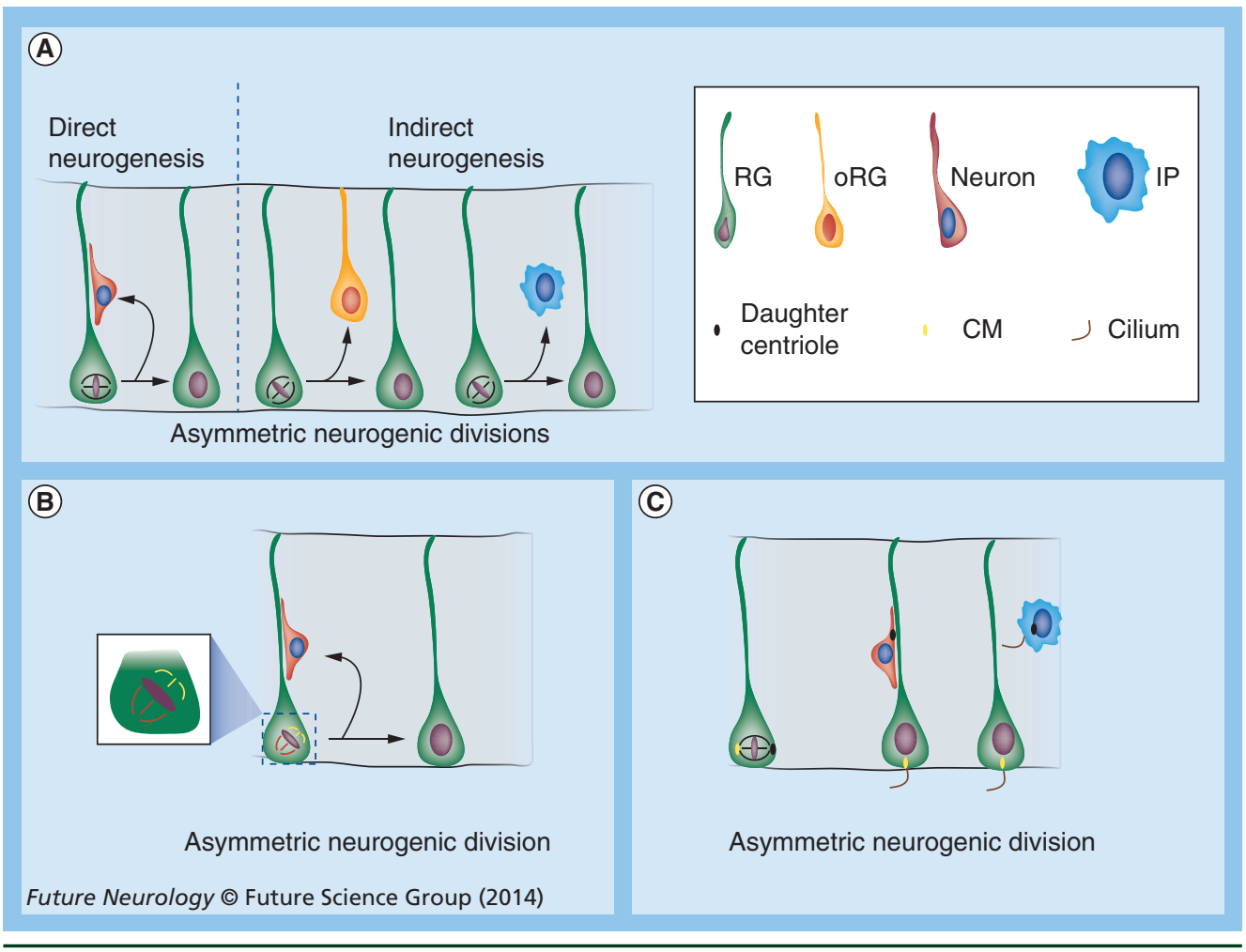

Figure 4. Mechanisms of asymmetric neurogenic progenitor divisions. (A) Orientation of the mitotic spindle appears to mediate direct or indirect (via IPs or oRGs) neurogenesis during asymmetric neurogenic radial glia progenitor (RGP) divisions. Oblique (or vertical, not illustrated) divisions preferentially give rise to neurons in an indirect manner. (B) Spindle size asymmetry correlates with the fate outcome of the daughter cells in asymmetric neurogenic RGP divisions. The daughter that originates from the larger spindle pole will become a neuron, while the other adopts a RGP fate. (C) Centrosome (i.e., centrioles) and CM inheritance could instruct daughter cell fates in asymmetric neurogenic RGP divisions. The cell that inherits the mother centriole associated with the ciliary membrane remnant becomes RGP, while the other adopts a neuron or IP fate.

CM: Ciliary membrane; IP: Intermediate progenitor; oRG: Outer radial glia; RG: Radial glia.

of IPs are present in the cortex: short radial apical IPs (with an apical process) in the VZ, and multipolar IPs in the SVZ (Figure 1) [88,89]. Both IP types derive from mitotic RGPs and their production is regulated by $\mathrm{Tbr} 2$ and cyclin D1/2 expression, RGP spindle orientation and cell cycle length $[38,53,77,84,90,91]$. Time lapse imaging in vivo has illustrated that apical IPs divide symmetrically to produce two neuron daughter cells. By contrast, IPs divide mostly symmetrically to generate two neurons but retain a limited capacity for self-renewal and thereby the potential to produce neuron doublets and/or quartets $[53,88]$. In vivo analysis of neurogenic IP divisions using the Tis21reporter, and loss of function of Tbr 2 in knockout mice indicated that IPs may contribute to the generation of pyramidal neurons of all cortical layers $[38,92,93]$.
SNPs are also located in the VZ [94,95] and can be distinguished from RGPs by tubulin $\alpha-1$ expression. SNPs contact the ventricular surface through a short apical process, which is retracted during mitosis. Interestingly, SNPs and RGPs have different cell cycle kinetics and contribute distinctly to overall cortical neurogenesis. While RGPs often generate neurons through IPs, SNPs produce postmitotic neurons (at least layer IV neurons during mid-neurogenesis) through symmetric neurogenic divisions [94,95].

\section{- Outer subventricular zone progenitors}

Cerebral cortex expansion, a trademark of mammalian brain evolution, is associated with an increase in neuron numbers [3,5,96,97]. However, a larger cortex size may not only reflect a higher number of neurons but instead also an extended variety of neuron and/or progenitor cell lineages. 
A new type of basally dividing progenitors (oSVZ progenitors or outer radial glia cells [oRGs]) has been recently identified in the oSVZ in humans [76], mice [77,78], ferret [98,99] and marmoset $[100,101]$. Unlike RGPs, oRGs are located far from the ventricle, with no apical contact to the luminal surface, but they possess a long basal process that extends to the pial surface [76]. Time lapse imaging of mice GFP-infected RGPs demonstrated that mice oRGs originate by asymmetric RGP division [78]. In the developing human neocortex oRGs are generated by oblique RGP divisions [102], consistent with the observation that mitotic spindle orientation alterations in dividing mouse RGPs (towards a more oblique mode of division) caused an increased oRG production $[77,84]$. Interestingly, dynamic live imaging revealed that oRGs undergo mitotic somal translocation although a functional significance has not yet been established [78]. Neurogenic oRGs produce neurons through asymmetric division but species-specific differences in oRG-mediated neurogenesis have been described. It appears that in the mouse, oRGs produce neurons directly and not via any IP [78]. By contrast, human oRGs typically divide asymmetrically, thereby selfrenewing and producing a bipolar daughter cell (TAP cell) capable of proceeding through multiple rounds of symmetric self-renewing divisions before expressing IP markers [76]. These findings may suggest that oRGs and/or TAPs and their unique neurogenic amplifying properties may account for the greater expansion and gyrification of the human neocortex $[5,96,97]$. This hypothesis was recently addressed by selective artificial amplification of either the IP or oRG progenitor pools in mice and ferrets $[103,104]$. Interestingly, it was found that IP amplification tends to promote tangential expansion of the cortex while an increased population of oRGs can trigger radial expansion and gyrification [103,104]. Radial expansion at sites of prospective gyra appears to correlate with decreased expression levels of the putative transcriptional regulator Trnp1 in developing human fetuses [104]. It will be intriguing to determine the entire spectrum of speciesspecific differences in oRG neurogenic potential. Interestingly, in primates (macaque monkey) five distinct types of oRG progenitors were identified in the oSVZ [105]. These different oRG classes may indeed exhibit distinct neurogenic capacities and thus contribute to generating neuronal diversity during neocortical development. Future analysis of species-specific qualitative and quantitative differences in the cortical oRG progenitor pools promises unprecedented insights into the evolutionary mechanisms imposing on neurogenesis.

\section{Conclusion \& future perspective}

The cerebral cortex is the largest structure of the human brain and composed of a sophisticated network of billions of neurons. It is not well understood how cortical neuronal circuits account for behavior and cognitive activity and how alterations in the cytoarchitecture may lead to neurological and psychiatric disorders or dementia. One approach, towards a more coherent mechanistic understanding, is to decipher the logic of neurogenesis and thus to determine the types and numbers of cortical neurons produced and how they successively build up the cortical entity during development. The cellular and molecular mechanisms controlling neurogenesis remain, however, still largely elusive and we here discuss a number of open key questions. Although several distinct classes of neuronal progenitors have been described $[5,6]$, it is still not clear how many distinct types effectively exist and may contribute qualitatively and quantitatively to cortical neurogenesis. Thus, it will be important to identify all progenitor types and determine the cellular and molecular principles that regulate the establishment of progenitor diversity, and how this in turn can influence the generation of distinct neurons. Along these lines, several models have been proposed regarding the multipotency of RGPs [30,44]. It has been recently suggested that fate-restricted RGPs exist albeit most experimental evidence indicates that RGPs are multipotent (i.e., produce all types of cortical projection neurons) and progressively restrict their developmental potential regarding the type of neuron that they produce at any given time $[30,44,63,106,107]$. To clarify the above issue it will be revealing to decipher the quantitative and qualitative clonal units on an individual progenitor level. Are the neurogenic potentials of distinct progenitors at any given developmental time predetermined, implicating a rather deterministic mode of neurogenesis? On a cell biological level, how does a progenitor know whether to divide symmetrically to expand its pool or start producing neurons? Once in the neurogenic division mode: what are the signaling pathways that in a progenitor direct the choice whether neurogenesis occurs directly or 
indirectly via IP and/or oRG? Lineage tracing approaches coupled with functional candidate gene analyses can help to contribute to our mechanistic understanding of the critical switch from expanding progenitor to asymmetric neurogenic divisions. On the circuit level, how does the lineage relationship of clonally related neurons derived from the same progenitor affect or instruct the specificity of neuronal connectivity in cortical circuits [108,109]? If we further project this question onto the stem and progenitor cells, one might ask whether distinct progenitors, and perhaps distinct progenitor lineages, encode prespecified neuron lineages, and

\section{EXECUTIVE SUMMARY}

\section{Overview: cerebral cortex development}

- The cerebral cortex is composed of an extraordinary number of projection and interneurons that originate from a diverse variety of neural stem cells, located in the ventricular zone (VZ)/subventricular zone (SVZ), during embryonic development.

- The precise cellular and molecular mechanisms regulating the functional neurogenic neural stem cell properties remain, to a large extent, elusive but disruptions in neurogenesis can lead to severe neurological disorders, including microcephaly, autism and epilepsy.

\section{Monitoring neurogenesis}

- Monitoring neurogenesis at high single progenitor cell resolution is essential for the quantitative and qualitative analysis of the neuronal lineages generated from individual classes of progenitors.

- Birth dating approaches using thymidine analogs or fluorescent transgenic probes, including Tis 21 and fluorescent, ubiquitination-based cell cycle indicator mice can reveal the precise cell cycle dynamics in proliferating progenitors.

- Sparse and/or conditional retrovirus-mediated progenitor cell infections can reveal radial glia progenitor (RGP) properties, such as morphology and cell division dynamics.

- Conditional transgenic reporter alleles in combination with TM-inducible CreER recombinases can sparsely mark proliferating progenitors and their lineages in a cell type-specific manner.

- Mosaic analysis with double markers can serve as a tool for lineage tracing experiments in real time and can be coupled to concomitant loss of gene function analysis.

\section{Cortical stem \& progenitor cells dynamically orchestrate cortical neurogenesis}

- Neuroepithelial cells are the earliest type of neural precursor in the neural tube and divide mostly symmetrically to amplify the progenitor pool.

- RGPs derive from neuroepithelial cells and initially divide mostly symmetrically to expand their pool. As the rate of neurogenesis increases, asymmetric neurogenic divisions dominate. Mitotic spindle orientation, size asymmetry and inheritance of mother centrosome, together with ciliary membrane, may regulate the outcome of asymmetric RGP divisions.

- Short neural precursors are located in the VZ and intermediate progenitors in the SVZ and contribute to neuron production.

- Outer radial glias derive from RGPs in the VZ by asymmetric oblique divisions. Expansion of intermediate progenitors and outer radial glias correlate with the expansion and gyrification of the neocortex during evolution.

\section{Future perspective}

- Future efforts shall reveal how many distinct progenitor types exist and how they each contribute qualitatively and quantitatively to cortical neurogenesis.

- Lineage tracing approaches coupled with functional candidate gene analyses will help to contribute to our mechanistic understanding of cortical neurogenesis.

- Determination of the precise mechanisms controlling neurogenesis may provide a basis for prospective future embryonic stem cell-based approaches in the context of directed brain repair. 
how different progenitors can, via their specific neuron output, contribute to shape the architecture of neuronal microcircuits in distinct functional cortical areas. Systematic monitoring of neurogenic parameters coupled with spatiotemporal lineage tracing and eventual physiological assessment of neuronal connectivity between clonally related neurons shall promise insights into the above issues. Lastly, how does the relative abundance of distinct progenitor cell types in different species drive the evolution of brain size and gyrencephaly? Progenitor types may have evolved new features and properties along the evolution of species. Thus, the systematic categorizing of progenitor types and their abundance in various species holds the promise of unprecedented insights not only into how progenitors build small or large brains in distinct species, but also into the underlying mechanisms shaping neocortex cytoarchitecture, morphology and gyrencephaly $[5,96,97]$. Lossof-function analyses of human specific candidate genes by using cerebral organoids may provide new insights also into the underlying

\section{References}

Papers of special note have been highlighted as:

- of interest; $\bullet$ of considerable interest

1 Abdel-Mannan O, Cheung AF, Molnar Z. Evolution of cortical neurogenesis. Brain Res. Bull. 75(2-4), 398-404 (2008).

2 Northcutt RG. Evolution of the telencephalon in nonmammals. Ann. Rev. Neurosci. 4, 301-350 (1981).

3 Geschwind DH, Rakic P. Cortical evolution: judge the brain by its cover. Neuron 80(3), 633-647 (2013).

4 Hill RS, Walsh CA. Molecular insights into human brain evolution. Nature 437(7055), 64-67 (2005).

5 Lui JH, Hansen DV, Kriegstein AR. Development and evolution of the human neocortex. Cell 146(1), 18-36 (2011).

6 Franco SJ, Muller U. Shaping our minds: stem and progenitor cell diversity in the mammalian neocortex. Neuron 77(1), 19-34 (2013).

7 His W. [The Neuroblasts and their Formation in Embryonic Nervous System]. S Hirzel Verlag, Stuttgart, Germany, 15 (1889).

8 Koelliker RA. [Handbook of Histology of Humans. Volume 2 Nervous System of Humans and Animals]. 6th Edition. Wilhelm Engelmann, Leipzig, Germany (1896).

basis of neurodevelopmental diseases including microcephaly [110].

In conclusion, future efforts aimed at indexing progenitor diversity and decoding their individual neurogenic properties should provide a framework to rigorously address the above open questions. In a broader context, these endeavors can also contribute to our knowledge of cortical neuron and microcircuit specification and thus provide a possible foundation for prospective future embryonic stem cell-based approaches in the context of directed brain repair [111,112].

\section{Acknowledgements}

The authors would like to thank $G$ Novarino for helpful comments on the manuscript and the Hippenmeyer laboratory members for discussions.

\section{Open access}

This work is licensed under the Creative Commons Attribution-NonCommercial 3.0 Unported License. To view a copy of this license, visit http://creativecommons.org/ licenses/by-nc-nd/3.0/

9 Schaper A. The earliest differentiation in the central nervous system of vertebrates. Science 5(115), 430-431 (1897).

10 Vignal W. Recherches sur le developpement des elements des couches corticales du cerveau et du cervelet chez l'homme et les mamiferes. Arch. Physiol. Norm. Path. (Paris) IV(2), 228-254 (1888).

11 Kosodo Y. Interkinetic nuclear migration: beyond a hallmark of neurogenesis. Cell. Mol. Life Sci. 69 (16), 2727-2738 (2012).

12 Lee HO, Norden C. Mechanisms controlling arrangements and movements of nuclei in pseudostratified epithelia. Trends Cell Biol. 23(3), 141-150 (2013).

13 Sauer FC. Mitoses in the neural tube. J. Comp. Neurol. 62, 377-405 (1935).

14 Malatesta P, Hartfuss E, Gotz M. Isolation of radial glial cells by fluorescent-activated cell sorting reveals a neuronal lineage. Development 127(24), 5253-5263 (2000).

15 Noctor SC, Flint AC, Weissman TA, Dammerman RS, Kriegstein AR. Neurons derived from radial glial cells establish radial units in neocortex. Nature 409(6821), $714-720$ (2001).

-. Uses a retroviral vector encoding enhanced GFP to label cortical progenitor cells in vivo. Using in vivo time-lapse recording, conclusively demonstrated for the first time that proliferative radial glia progenitors
(RGPs) generate neurons in the developing cortex.

16 Angevine JB Jr, Sidman RL. Autoradiographic study of cell migration during histogenesis of cerebral cortex in the mouse. Nature 192, 766-768 (1961).

17 Rakic P. Neurons in rhesus monkey visual cortex: systematic relation between time of origin and eventual disposition. Science 183(4123), 425-427 (1974).

18 Geschwind DH. Genetics of autism spectrum disorders. Trends Cogn. Sci. 15(9), 409-416 (2011).

19 Manzini MC, Walsh CA. What disorders of cortical development tell us about the cortex: one plus one does not always make two. Curr. Opin. Genet. Devel. 21(3), 333-339 (2011).

20 Reiner O. LIS1 and DCX: implications for brain development and human disease in relation to microtubules. Scientifica 2013, 393975 (2013).

21 Ross ME, Walsh CA. Human brain malformations and their lessons for neuronal migration. Ann. Rev. Neurosci. 24, 1041-1070 (2001).

22 Wynshaw-Boris A, Pramparo T, Youn YH, Hirotsune S. Lissencephaly: mechanistic insights from animal models and potential therapeutic strategies. Semin. Cell Devel. Biol. 21(8), 823-830 (2010). 
23 Kepecs A, Fishell G. Interneuron cell types are fit to function. Nature 505(7483), 318-326 (2014).

24 Evsyukova I, Plestant C, Anton ES. Integrative mechanisms of oriented neuronal migration in the developing brain. Ann. Rev. Cell Devel. Biol. 29, 299-353 (2013).

25 Marin O. Cellular and molecular mechanisms controlling the migration of neocortical interneurons. Eur. J. Neurosci. 38(1), 2019-2029 (2013).

26 Metin C, Vallee RB, Rakic P, Bhide PG Modes and mishaps of neuronal migration in the mammalian brain. J. Neurosci. 28(46), 11746-11752 (2008).

27 Anthony TE, Klein C, Fishell G, Heintz N. Radial glia serve as neuronal progenitors in all regions of the central nervous system. Neuron 41(6), 881-890 (2004)

28 Brown KN, Chen S, Han Z et al. Clonal production and organization of inhibitory interneurons in the neocortex. Science 334(6055), 480-486 (2011).

- Combined conditional Cre-mediated recombination with sparse retroviral infections of medial ganglionic eminence RGP progenitors generating cortical interneurons.

29 Ciceri G, Dehorter N, Sols I, Huang ZJ, Maravall M, Marin O. Lineage-specific laminar organization of cortical GABAergic interneurons. Nat. Neurosci. 16(9), 1199-1210 (2013).

- Combined conditional Cre-mediated recombination with sparse retroviral infections of medial ganglionic eminence RGP progenitors generating cortical interneurons.

30 Greig LC, Woodworth MB, Galazo MJ, Padmanabhan H, Macklis JD. Molecular logic of neocortical projection neuron specification, development and diversity. Nat. Rev. Neurosci. 14(11), 755-769 (2013).

31 Kessaris N, Magno L, Rubin AN, Oliveira MG. Genetic programs controlling cortical interneuron fate. Curr. Opin. Neurobiol. 26C, 79-87 (2014).

32 Farkas LM, Huttner WB. The cell biology of neural stem and progenitor cells and its significance for their proliferation versus differentiation during mammalian brain development. Curr. Opin. Cell Biol. 20(6), 707-715 (2008)

33 Sidman RL, Miale IL, Feder N. Cell proliferation and migration in the primitive ependymal zone: an autoradiographic study of histogenesis in the nervous system. Exp. Neurol. 1, 322-333 (1959).
34 Aten JA, Bakker PJ, Stap J, Boschman GA, Veenhof $\mathrm{CH}$. DNA double labelling with IdUrd and CldUrd for spatial and temporal analysis of cell proliferation and DNA replication. Histochem. J. 24(5), 251-259 (1992).

35 Gratzner HG. Monoclonal antibody to 5-bromo- and 5-iododeoxyuridine: a new reagent for detection of DNA replication. Science 218(4571), 474-475 (1982).

36 Nowakowski RS, Lewin SB, Miller MW. Bromodeoxyuridine immunohistochemical determination of the lengths of the cell cycle and the DNA-synthetic phase for an anatomically defined population. J. Neurocytol. 18(3), 311-318 (1989).

37 Livesey FJ, Cepko CL. Vertebrate neural cell-fate determination: lessons from the retina. Nat. Rev. Neurosci. 2(2), 109-118 (2001).

38 Haubensak W, Attardo A, Denk W, Huttner WB. Neurons arise in the basal neuroepithelium of the early mammalian telencephalon: a major site of neurogenesis. Proc. Natl Acad. Sci. USA 101(9), 3196-3201 (2004).

39 Arai Y, Pulvers JN, Haffner C et al. Neural stem and progenitor cells shorten S-phase on commitment to neuron production. Nat. Commun. 2, 154 (2011).

40 Calegari F, Haubensak W, Haffner C, Huttner WB. Selective lengthening of the cell cycle in the neurogenic subpopulation of neural progenitor cells during mouse brain development. J. Neurosci. 25(28), 6533-6538 (2005).

41 Sakaue-Sawano A, Kurokawa H, Morimura T et al. Visualizing spatiotemporal dynamics of multicellular cell-cycle progression. Cell 132(3), 487-498 (2008).

- Describes a unique technique, fluorescent ubiquitination-based cell cycle indicator, designed to specifically track cell cycle transitions in living cells with high spatiotemporal resolution. Fluorescent ubiquitination-based cell cycle indicator offers the possibility to gain new insights into regulation of progenitor cell cycle progression.

42 Soriano P. Generalized lacZ expression with the ROSA26 Cre reporter strain. Nat. Genet. 21(1), 70-71 (1999).

43 Abe T, Sakaue-Sawano A, Kiyonari $\mathrm{H}$ et al. Visualization of cell cycle in mouse embryos with Fucci2 reporter directed by Rosa26 promoter. Development 140(1), 237-246 (2013).

44 Bartolini G, Ciceri G, Marin O. Integration of GABAergic interneurons into cortical cell assemblies: lessons from embryos and adults. Neuron 79(5), 849-864 (2013).

45 Blanpain C, Simons BD. Unravelling stem cell dynamics by lineage tracing. Nat. Rev. Mol. Cell Biol. 14(8), 489-502 (2013).

46 Buckingham ME, Meilhac SM. Tracing cells for tracking cell lineage and clonal behavior. Dev. Cell 21(3), 394-409 (2011).

47 Kretzschmar K, Watt FM. Lineage tracing. Cell 148(1-2), 33-45 (2012).

48 Legue E, Joyner AL. Genetic fate mapping using site-specific recombinases. Methods Enzymol. 477, 153-181 (2010).

49 Cepko CL, Austin CP, Walsh C, Ryder EF, Halliday A, Fields-Berry S. Studies of cortical development using retrovirus vectors. Cold Spring Harb. Symp. Quant. Biol. 55, 265-278 (1990).

50 Sanes JR. Analysing cell lineage with a recombinant retrovirus. Trends Neurosci. 12(1), 21-28 (1989)

51 Walsh C, Cepko CL. Widespread dispersion of neuronal clones across functional regions of the cerebral cortex. Science 255(5043), 434-440 (1992).

52 Noctor SC. Time-lapse imaging of fluorescently labeled live cells in the embryonic mammalian forebrain. Cold Spring Harb. Prot. 2011(11), 1350-1361 (2011).

53 Noctor SC, Martinez-Cerdeno V, Ivic L, Kriegstein AR. Cortical neurons arise in symmetric and asymmetric division zones and migrate through specific phases. Nat. Neurosci. 7(2), 136-144 (2004).

54 Tsai JW, Vallee RB. Live microscopy of neural stem cell migration in brain slices. Methods Mol. Biol. 750, 131-142 (2011).

55 Tsai JW, Chen Y, Kriegstein AR, Vallee RB. LIS1 RNA interference blocks neural stem cell division, morphogenesis, and motility at multiple stages. J. Cell Biol. 170(6), 935-945 (2005).

56 Hippenmeyer S, Vrieseling E, Sigrist M et al. A developmental switch in the response of DRG neurons to ETS transcription factor signaling. PLoS Biol. 3(5), e159 (2005).

57 Madisen L, Zwingman TA, Sunkin SM et al. A robust and high-throughput Cre reporting and characterization system for the whole mouse brain. Nat. Neurosci. 13(1), 133-140 (2010).

58 Novak A, Guo C, Yang W, Nagy A, Lobe CG. Z/EG, a double reporter mouse line that expresses enhanced green fluorescent protein upon Cre-mediated excision. Genesis 28(3-4), 147-155 (2000).

59 Kratochwil CF, Rijli FM. The Cre/Lox system to assess the development of the mouse 
brain. Methods Mol. Biol. 1082, 295-313 (2014).

60 Metzger D, Chambon P. Site- and timespecific gene targeting in the mouse. Methods 24(1), 71-80 (2001).

61 Livet J, Weissman TA, Kang $\mathrm{H}$ et al. Transgenic strategies for combinatorial expression of fluorescent proteins in the nervous system. Nature 450(7166), 56-62 (2007).

62 Snippert HJ, Van Der Flier LG, Sato T et al. Intestinal crypt homeostasis results from neutral competition between symmetrically dividing Lgr5 stem cells. Cell 143(1), 134-144 (2010)

63 Guo C, Eckler MJ, Mckenna WL, Mckinsey GL, Rubenstein JL, Chen B. Fezf2 expression identifies a multipotent progenitor for neocortical projection neurons, astrocytes, and oligodendrocytes. Neuron 80(5), 1167-1174 (2013)

64 Hippenmeyer S. Dissection of gene function at clonal level using mosaic analysis with double markers. Front Biol. 8(6), 557-568 (2013).

65 Jefferis GS, Livet J. Sparse and combinatorial neuron labelling. Curr. Opin. Neurobiol. 22(1), 101-110 (2012).

66 Luo L. Fly MARCM and mouse MADM: genetic methods of labeling and manipulating single neurons. Brain Res. Rev. 55(2), 220-227 (2007).

67 Zong H, Espinosa JS, Su HH, Muzumdar MD, Luo L. Mosaic analysis with double markers in mice. Cell 121(3), 479-492 (2005).

- Describes mosaic analysis with double markers (MADM) that allows the generation of genetic mosaics in mice whereby wild-type and mutant cell clones are uniquely labeled. Together with [69], these studies illustrate the potential of MADM for high-resolution lineage tracing in vivo.

68 Hippenmeyer S, Johnson RL, Luo L. Mosaic analysis with double markers reveals cell-type-specific paternal growth dominance. Cell Reports 3(3), 960-967 (2013).

69 Hippenmeyer S, Youn YH, Moon HM et al. Genetic mosaic dissection of Lis1 and Ndell in neuronal migration. Neuron 68(4), 695-709 (2010)

-- Describes MADM that allows the generation of genetic mosaics in mice whereby wild-type and mutant cell clones are uniquely labeled. Together with [67], these studies illustrate the potential of MADM for high-resolution lineage tracing in vivo.
70 Shitamukai A, Matsuzaki F. Control of asymmetric cell division of mammalian neural progenitors. Dev. Growth Differ. 54(3), 277-286 (2012).

71 Yingling J, Youn YH, Darling D et al. Neuroepithelial stem cell proliferation requires LIS1 for precise spindle orientation and symmetric division. Cell 132(3), 474-486 (2008).

72 Mccarthy M, Turnbull DH, Walsh CA, Fishell G. Telencephalic neural progenitors appear to be restricted to regional and glial fates before the onset of neurogenesis. J. Neurosci. 21(17), 6772-6781 (2001).

73 Qian X, Shen Q, Goderie SK et al. Timing of CNS cell generation: a programmed sequence of neuron and glial cell production from isolated murine cortical stem cells. Neuron 28(1), 69-80 (2000).

74 Williams BP, Price J. Evidence for multiple precursor cell types in the embryonic rat cerebral cortex. Neuron 14(6), 1181-1188 (1995).

75 Gotz M, Huttner WB. The cell biology of neurogenesis. Nat. Rev. Mol. Cell Biol. 6(10), 777-788 (2005)

76 Hansen DV, Lui JH, Parker PR, Kriegstein AR. Neurogenic radial glia in the outer subventricular zone of human neocortex. Nature 464(7288), 554-561 (2010).

77 Shitamukai A, Konno D, Matsuzaki F. Oblique radial glial divisions in the developing mouse neocortex induce self-renewing progenitors outside the germinal zone that resemble primate outer subventricular zone progenitors. J. Neurosci. 31(10), 3683-3695 (2011).

78 Wang X, Tsai JW, Lamonica B, Kriegstein AR. A new subtype of progenitor cell in the mouse embryonic neocortex. Nat. Neurosci. 14(5), 555-561 (2011).

79 Delaunay D, Cortay V, Patti D, Knoblauch K, Dehay C. Mitotic spindle asymmetry: a Wnt/PCP-regulated mechanism generating asymmetrical division in cortical precursors. Cell Reports 6(2), 400-414 (2014).

80 Knoblich JA. Mechanisms of asymmetric stem cell division. Cell 132(4), 583-597 (2008).

81 Paridaen JT, Wilsch-Brauninger M, Huttner WB. Asymmetric inheritance of centrosomeassociated primary cilium membrane directs ciliogenesis after cell division. Cell 155(2), 333-344 (2013).

82 Tan X, Shi SH. Neocortical neurogenesis and neuronal migration. Wiley Interdiscip. Rev. Dev. Biol. 2(4), 443-459 (2013).

83 Xie Y, Juschke C, Esk C, Hirotsune S, Knoblich JA. The phosphatase PP4c controls spindle orientation to maintain proliferative symmetric divisions in the developing neocortex. Neuron 79 (2), 254-265 (2013).

Postiglione MP, Juschke C, Xie Y, Haas GA, Charalambous C, Knoblich JA. Mouse inscuteable induces apical-basal spindle orientation to facilitate intermediate progenitor generation in the developing neocortex. Neuron 72(2), 269-284 (2011).

- Demonstrates that mouse INSC-mediated control of cleavage plane orientation in mitotic RGPs regulates their output. Oblique RGP cleavage planes preferentially promote the generation of basal progenitors (intermediate progenitors and outer radial glia $[o R G]$ )

85 Wang X, Tsai JW, Imai JH, Lian WN, Vallee $\mathrm{RB}$, Shi SH. Asymmetric centrosome inheritance maintains neural progenitors in the neocortex. Nature 461(7266), 947-955 (2009).

86 Lehtinen MK, Walsh CA. Neurogenesis at the brain-cerebrospinal fluid interface. Ann. Rev. Cell Devel. Biol. 27, 653-679 (2011).

87 Englund C, Fink A, Lau C et al. Pax6, Tbr2, and $\mathrm{Tbr} 1$ are expressed sequentially by radial glia, intermediate progenitor cells, and postmitotic neurons in developing neocortex. J. Neurosci. 25(1), 247-251 (2005).

88 Kowalczyk T, Pontious A, Englund C et al. Intermediate neuronal progenitors (basal progenitors) produce pyramidal-projection neurons for all layers of cerebral cortex. Cerebral cortex 19(10), 2439-2450 (2009).

89 Kawaguchi A, Ikawa T, Kasukawa T et al. Single-cell gene profiling defines differential progenitor subclasses in mammalian neurogenesis. Development 135(18), 3113-3124 (2008).

90 Glickstein SB, Monaghan JA, Koeller HB, Jones TK, Ross ME. Cyclin D2 is critical for intermediate progenitor cell proliferation in the embryonic cortex. J. Neurosci. 29(30), 9614-9624 (2009).

91 Lange C, Huttner WB, Calegari F. Cdk4/ cyclinD1 overexpression in neural stem cells shortens G1, delays neurogenesis, and promotes the generation and expansion of basal progenitors. Cell Stem Cell 5(3) 320-331 (2009).

92 Arnold SJ, Huang GJ, Cheung AF et al. The T-box transcription factor Eomes/Tbr2 regulates neurogenesis in the cortical subventricular zone. Genes Devel. 22(18), 2479-2484 (2008).

93 Sessa A, Mao CA, Hadjantonakis AK, Klein WH, Broccoli V. Tbr2 directs conversion of radial glia into basal precursors and guides 
neuronal amplification by indirect neurogenesis in the developing neocortex. Neuron 60 (1), 56-69 (2008).

94 Gal JS, Morozov YM, Ayoub AE, Chatterjee M, Rakic P, Haydar TF. Molecular and morphological heterogeneity of neural precursors in the mouse neocortical proliferative zones. J. Neurosci. 26(3), 1045-1056 (2006).

95 Stancik EK, Navarro-Quiroga I, Sellke R, Haydar TF. Heterogeneity in ventricular zone neural precursors contributes to neuronal fate diversity in the postnatal neocortex. J. Neurosci. 30(20), 7028-7036 (2010).

96 Borrell V, Reillo I. Emerging roles of neural stem cells in cerebral cortex development and evolution. Devel. Neurobiol. 72(7), 955-971 (2012).

97 Fietz SA, Huttner WB. Cortical progenitor expansion, self-renewal and neurogenesis - a polarized perspective. Curr. Opin. Neurobiol. 21(1), 23-35 (2011).

98 Fietz SA, Kelava I, Vogt J et al. OSVZ progenitors of human and ferret neocortex are epithelial-like and expand by integrin signaling. Nat. Neurosci. 13(6), 690-699 (2010).

99 Reillo I, De Juan Romero C, Garcia-Cabezas MA, Borrell V. A role for intermediate radial glia in the tangential expansion of the mammalian cerebral cortex. Cereb. Cortex 21(7), 1674-1694 (2011).

100 Garcia-Moreno F, Vasistha NA, Trevia N, Bourne JA, Molnar Z. Compartmentalization of cerebral cortical germinal zones in a lissencephalic primate and gyrencephalic rodent. Cereb. Cortex 22 (2), 482-492 (2012).

101 Kelava I, Reillo I, Murayama AY et al. Abundant occurrence of basal radial glia in the subventricular zone of embryonic neocortex of a lissencephalic primate, the common marmoset Callithrix jacchus. Cereb. Cortex 22(2), 469-481 (2012).

102 Lamonica BE, Lui JH, Hansen DV, Kriegstein AR. Mitotic spindle orientation predicts outer radial glial cell generation in human neocortex. Nat. Commun. 4, 1665 (2013).

103 Nonaka-Kinoshita M, Reillo I, Artegiani B et al. Regulation of cerebral cortex size and folding by expansion of basal progenitors. EMBO J. 32(13), 1817-1828 (2013).

- Shows that expansion of the oRG pool in the ferret brain triggers the formation of novel cortical folds (gyrification). Together with [104], suggests that an increase in the number of oRGs may represent a key evolutionary event promoting gyrification in the mammalian brain.

104 Stahl R, Walcher T, De Juan Romero C et al. Trnp1 regulates expansion and folding of the mammalian cerebral cortex by control of radial glial fate. Cell 153(3), 535-549 (2013).

- Shows that expansion of the oRG pool in the ferret brain triggers the formation of novel cortical folds (gyrification). This paper, together with [103], suggests that an increase in the number of oRGs may represent a key evolutionary event promoting gyrification in the mammalian brain.
105 Betizeau M, Cortay V, Patti D et al. Precursor diversity and complexity of lineage relationships in the outer subventricular zone of the primate. Neuron 80(2), 442-457 (2013).

106 Franco SJ, Gil-Sanz C, Martinez-Garay I et al. Fate-restricted neural progenitors in the mammalian cerebral cortex. Science 337(6095), 746-749 (2012).

107 Kuan CY, Elliott EA, Flavell RA, Rakic P. Restrictive clonal allocation in the chimeric mouse brain. Proc. Natl Acad. Sci. USA 94(7), 3374-3379 (1997).

108 Gao P, Sultan KT, Zhang XJ, Shi SH. Lineage-dependent circuit assembly in the neocortex. Development 140(13), 2645-2655 (2013).

109 Smith GB, Fitzpatrick D. Specifying cortical circuits: a role for cell lineage. Neuron 75(1), 4-5 (2012).

110 Lancaster MA, Renner M, Martin CA et al. Cerebral organoids model human brain development and microcephaly. Nature 501(7467), 373-379 (2013).

111 Espuny-Camacho I, Michelsen KA, Gall D et al. Pyramidal neurons derived from human pluripotent stem cells integrate efficiently into mouse brain circuits in vivo. Neuron 77(3), 440-456 (2013).

112 Gaspard N, Vanderhaeghen P. From stem cells to neural networks: recent advances and perspectives for neurodevelopmental disorders. Dev. Med. Child Neurol. 53(1), 13-17 (2011). 


\section{Monitoring neurogenesis in the cerebral cortex: an update}

To obtain credit, you should first read the journal article. After reading the article, you should be able to answer the following, related, multiple-choice questions. To complete the questions (with a minimum $70 \%$ passing score) and earn continuing medical education (CME) credit, please go to www.medscape.org/journal/fnl. Credit cannot be obtained for tests completed on paper, although you may use the worksheet below to keep a record of your answers. You must be a registered user on Medscape.org. If you are not registered on Medscape.org, please click on the New Users: Free Registration link on the left hand side of the website to register. Only one answer is correct for each question. Once you successfully answer all post-test questions you will be able to view and/or print your certificate. For questions regarding the content of this activity, contact the accredited provider,
CME@medscape.net. For technical assistance, contact CME@webmd.net. American Medical Association's Physician's Recognition Award (AMA PRA) credits are accepted in the US as evidence of participation in CME activities. For further information on this award, please refer to http://www.ama-assn.org/ama/pub/ category/2922.html. The AMA has determined that physicians not licensed in the US who participate in this CME activity are eligible for AMA PRA Category 1 Credits $^{\mathrm{TM}}$. Through agreements that the AMA has made with agencies in some countries, AMA PRA credit may be acceptable as evidence of participation in CME activities. If you are not licensed in the US, please complete the questions online, print the AMA PRA CME credit certificate and present it to your national medical association for review.

\section{Activity evaluation: where 1 is strongly disagree and $\mathbf{5}$ is strongly agree.}

The activity supported the learning objectives.

The material was organized clearly for learning to occur.

The content learned from this activity will impact my practice.

The activity was presented objectively and free of commercial bias.

1. According to the review by Drs. Postiglione and Hippenmeyer, which of the following statements about recent techniques for monitoring neurogenesis is correct?
A Birth-dating approaches using guanidine analogues can reveal precise cell cycle dynamics in proliferating progenitors
B Sparse and/or conditional progenitor cell infections mediated by DNA viruses can reveal properties of radial glia progenitors (RGPS)
C Tamoxifen does not affect CreER recombinases
D Mosaic analysis with double markers can be a tool for lineage-tracing experiments in real time and can be coupled to concomitant loss of gene function analysis

2. According to the review by Drs. Postiglione and Hippenmeyer, which of the following statements about recent advances in understanding the dynamics of neurogenesis is correct?
A Neuroepithelial cells (NECS) appear relatively late in neurogenesis
B RGPS give rise to NECS
C Short neural precursors in the ventricular zone contribute to neuron production
D Outer radial glial cells give rise to RGPs in the subventricular zone 
3. According to the review by Drs. Postiglione and Hippenmeyer, which of the following statements about future directions for research and clinical implications regarding neurogenesis would most likely be correct?

$\square$ A It is well understood how alterations in the cytoarchitecture may lead to neurologic and psychiatric disorders or dementia

B No evidence to date exists that RGPs are multipotent

C Functional candidate gene analyses are unlikely to be helpful in understanding neurogenesis

D Assessing progenitor diversity and individual neurogenic properties could provide a possible foundation for prospective future embryonic stem cell-based approaches to directed brain repair 